\title{
Generalized Commuting Graph of Dihedral, Semi-dihedral and Quasi-dihedral Groups
}

\author{
Mustafa Anis El-Sanfaz ${ }^{a}$, Nor Haniza Sarmin ${ }^{b}$, Siti Norziahidayu Amzee \\ Zamric,*
}

a Mathematics Program, Department of Mathematics, Statistics and Physics, College of Arts and Sciences, Qatar University, 2713, Doha, Qatar; b Department of Mathematical Sciences, Faculty of Science, Universiti Teknologi Malaysia, 81310 UTM Johor Bahru, Johor, Malaysia; ' UniSZA Science and Medicine Foundation Centre, Universiti Sultan Zainal Abidin, Gong Badak Campus, 21300 Kuala Nerus, Terengganu

Abstract Commuting graphs are characterized by vertices that are non-central elements of a group where two vertices are adjacent when they commute. In this paper, the concept of commuting graph is extended by defining the generalized commuting graph. Furthermore, the generalized commuting graph of the dihedral groups, the quasi-dihedral groups and the semidihedral groups are presented and discussed. The graph properties including chromatic and clique numbers are also explored.

Keywords: graph theory, commuting graph, dihedral group, semi-dihedral group, quasi-dihedral group.

\section{Introduction}

In this section, some basic concepts of graph theory are explained.

A graph $\Gamma$, consists of edges and vertices, where symbol $V(\Gamma)$ represent vertices and $E(\Gamma)$ indicate the edges [1]. In addition, if the edges pass ordered pair of vertices, such a graph is referred to as a directed graph; if not, it's an undirected graph. Moreover, when an edge links two vertices, they are adjacent [2]. When a path exists between any two random vertices, it is a connected graph, otherwise it is a disconnected graph [3]. Meanwhile, when all vertices are connected to each other a complete graph is formed, denoted by the symbol $K_{n}$ [4]. Next, graphs with no vertices are defined as null graphs and denoted by $K_{0}$, while empty graphs has no edges between its vertices, denoted by $K_{\varepsilon}$ [2].

*For correspondence:

sitinamzee@unisza.edu.my

Received: 25 May 2021

Accepted: 24 Oct 2021

(C) Copyright El-Sanfaz et al. This article is distributed under the terms of the Creative Commons

Attribution License, which permits unrestricted use and redistribution provided that the original author and source are credited.

The idea of commuting graphs is presented from the commuting elements that do not belong to the centre of a group. In [5], commuting graphs are characterized by vertices that are non-central elements of a group where two vertices are adjacent when they commute. Research on commuting graph is fairly a recent phenomenon, which has developed in various contexts.

In 2001, Segev [6] determined the commuting graph of non-solvable groups. In 2006, Bundy [7] studied the connectivity of commuting graph where he determined a necessary and sufficient condition for the connectivity of commuting graphs of the symmetric group of degree $n$. In 2008, Iranmanesh and Jafarzadeh [8] investigated the properties of commuting graph of symmetric groups and alternating groups. They conjectured that if $n$ is a finite group and its commuting graph is connected, then the diameter of the commuting graph of $n$ is less than or equal to an arbitrary natural number. In 2009, Bates et al. [9] found certain properties of commuting graphs of the symmetric group of degree $n$. The diameter 
and disc structure of this graph were also investigated. In 2011, Chelvam et al. [10] discussed the commuting graph of dihedral groups. In addition, Raza and Faizi's results [11] showed the chromatic and clique number of the dihedral group of order $2 n$. In 2013, Parker [12] investigated the commuting graph of a finite soluble group with trivial centre. In addition, he has shown that the diameter of such a graph is at most 8 or the graph is disconnected. In the same year, Azimi et al. [13] introduced Jacobson graph of commuting rings where they expanded the research on its isomorphism [14], cycles and paths [15], as well as found that all Jacobson graphs have a matching that misses at most one vertex and offer 1-factor decomposition of a regular subgraph [16]. Additionally, in 2015, Akbari et al. [17] studied inclusion finite graph of some finite groups. In 2016, Das et al. [18] determine up to isomorphism all finite non-abelian groups whose commuting graphs are cyclic, planar or toroidal. Moreover, they derived explicit formulas for the genus of the commuting graphs of some well-known class of finite non-abelian groups, and they show that every collection of isomorphism classes of finite non-abelian groups whose commuting graphs have the same genus is finite. Following that, Zaid et al. [19-21] have connected some commutativity degree with generalized conjugacy class graph, and characterize some finite rings of matrices where their zero divisor graphs are also found in 2020. Recently, square graphs of some finite groups have also been determined [22].

This paper is structured as follows: Section 1 provided some fundamental concepts of graph theory. In Section 2, the methodology of this research will be explained. The results and discussions will be presented in Section 3, followed by conclusion in Section 4.

\section{Methodology of the research}

In this section, the methodology of this research is presented. This research started with the study on the fundamental concepts in graph theory, which include different types of graphs and their properties. However, the focus of this research is on the commuting graph, presented in the following.

Definition 1 [23] Commuting Graph

Let $G$ be a finite non-abelian group with the centre denoted by $Z(G)$. A commuting graph is a graph whose vertices are non-central elements of $G$ i.e. $G \backslash Z(G)$. Two vertices $v_{1}$ and $v_{2}$ are adjacent whenever $v_{1} v_{2}=v_{2} v_{1}$.

Later on, the commuting graph is extended where a new graph named as the generalized commuting graph is introduced, defined in the following.

\section{Definition 2 The Generalized Commuting Graph}

Suppose $G$ is a finite non-abelian group, and $\Omega$ a non-empty subset of $G \times G$. The generalized commuting graph $\Gamma_{\Omega}^{G C}$ is a graph whose vertices are non-central elements of $\Omega$ in $G$ i.e. $V\left(\Gamma_{\Omega}^{G C}\right)=\Omega-A$, where $A=\{\omega \in \Omega: g \omega=\omega g: g \in G\}$. Two vertices $\omega_{1}, \omega_{2} \in \Omega$ are adjacent if $\omega_{1} \omega_{2}=\omega_{2} \omega_{1}$, where $\Omega=\{(a, b) \in G \times G:|a|=|b|=2, a b=b a, a \neq b\}$.

In addition, the study on the commutativity degree, which is the probability that two elements in a group selected at random commute with each other was also conducted. It is found that the commuting operation has been widely used in group theory, which has motivated several researchers to associate this idea with graph theory.

Therefore, several results on the commutativity degree, named as the probability that an element of a group fixes a set by conjugation are referred. However, only three groups, namely the dihedral groups, semi-dihedral groups and quasi-dihedral groups are considered. Since the focus of this research is on three groups, their definitions are given in the following.

Definition 3 [24] Dihedral Group

The dihedral group of order $2 n$ is a group generated by two elements $a$ and $b$. The presentation of this 
group is expressed as $D_{n}:\left\langle a, b: d^{n}=b^{2}=1, a b=b a^{-1}\right\rangle$.

Definition 4 [24] Semi-dihedral Group

The semi-dihedral group is a group generated by two elements $a$ and $b$ such that $S D \cong$ $\left\langle a, b: a^{2 n}=b^{2}=e, a b=b a^{2^{n-1}-1}\right\rangle$, where $n \geq 3$.

Definition 5 [24] Quasi-Dihedral Group

The quasi-dihedral group is a group generated by two elements $a$ and $b$ such that $Q D \cong$ $\left\langle a, b: a^{2 n}=b^{2}=e, a b=b a^{2^{n-1}+1}\right\rangle$, where $n \geq 3$.

Next, the probability that an element of a group fixes a set by conjugation of these three groups are presented in the following three theorems.

Theorem 1 [23] Let $G$ be a dihedral group of order $2 n$ such that $G \cong\left\langle a, b: a^{n}=b^{2}=1, a b=b a^{-1}\right\rangle$. If $G$ acts on $G$ by conjugation, then $|\Omega|=3\left(\frac{n}{2}\right)$ represented as follows: There are $n$ elements in the form of $\left(a^{\frac{n}{2}}, a^{\frac{n}{2}+i} b\right), 0 \leq i \leq 2 n$ and $\frac{n}{2}$ are in the form of $\left(a^{i} b, a^{\frac{n}{2}+i} b\right), 0 \leq i \leq 2 n$.

Theorem 2 [25] Let $G$ be a semi-dihedral group, $G \cong\left\langle a, b: a^{2^{n}}=b^{2}=e, a b=b a^{2^{n-1}-1}\right\rangle$ where $n \geq 3$. If $G$ acts on $\Omega$ by conjugation, then $|\Omega|=3\left(2^{n-2}\right)$, represented as follows: There are $2^{n-1}$ elements in the form of $\left(a^{2^{n-1}}, a^{i} b\right), 0 \leq i \leq 2^{n}$ where $i$ is even and there are $2^{n-2}$ elements in the form of $\left(a^{i} b, a^{i+2^{n-1}} b\right), 0 \leq i \leq 2^{n}$ where $i$ is even.

Theorem 3 [25] Let $G$ be a quasi-dihedral group, $G \cong\left\langle a, b: a^{2^{n}}=b^{2}=e, a b=b a^{2^{n-1}+1}\right\rangle$ where $n \geq 3$. If $G$ acts on $\Omega$ by conjugation, then $|\Omega|=3$, given as follows: There are two elements in the form of $\left(a^{2^{n-1}}, a^{2^{n-1} i} b\right), 0 \leq i \leq 2^{n}$, and there is only one element in the form of $\left(a^{2^{n-1}} b, b\right)$.

In the next section, the results of these probabilities will be associated with the newly introduced generalized commuting graph, where several theorems and examples are given.

\section{Results and discussion}

This section presents our main results. First, some general results on the generalized commuting graph are provided., followed by the results on the computation of the generalized commuting graphs of the dihedral group, the semi-dihedral group and the quasi-dihedral group.

\section{Generalized commuting graph of finite group}

In this section, results on the generalized commuting graph are given. As the generalized commuting graph has been defined in previous section, the following results indicate some properties of the generalized commuting graph.

Proposition 1: If $G$ is an Abelian group, then $\Gamma_{\Omega}^{G C}$ is a null graph.

Proof: Since all elements of $G$ are in the center of the group, based on Definition $2,|\Omega|=|A|$. It follows that $\left|V\left(\Gamma_{\Omega}^{G C}\right)\right|=0$, thus $\Gamma_{\Omega}^{G C}$ is null. 
Generalized commuting graphs of dihedral groups, semi-dihedral groups and quasi-dihedral groups

In this section, generalized commuting graphs of the dihedral group, the semi-dihedral group and the quasi-dihedral group are presented.

In the following theorem, the generalized commuting graph is found for the dihedral group.

Theorem 4: Let $G$ be a dihedral group of order $2 n$ where $n$ is even. Let $\Omega$ be a non-empty subset of $G \times G$. Then $\Gamma_{\Omega}^{G C}=\bigcup_{i=1}^{\frac{n}{2}} K_{3}$.

Proof: Recall from Theorem 1, $|\Omega|=\frac{3 n}{2}$. Based on Definition 2, the number of vertices in $\Gamma_{\Omega}^{G C}$ is $\frac{3 n}{2}$ i.e. $\left|V\left(\Gamma_{\Omega}^{G C}\right)\right|=\frac{3 n}{2}$. Now, two vertices $\omega_{1}, \omega_{2}$ are adjacent if $\omega_{1} \omega_{2}=\omega_{2} \omega_{1}$. It follows that, the vertices which are in the form of $\left(a^{i} b, a^{\frac{n}{2}+i} b\right)\left(a^{i} b, a^{\frac{n}{2}}\right), 0 \leq i \leq 2 n$ are adjacent. The same is true for the vertices which are in the form of $\left(a^{i} b, a^{\frac{n}{2}+i} b\right)\left(a^{\frac{n}{2}+i} b, a^{\frac{n}{2}}\right), 0 \leq i \leq 2 n$. Since $a^{i} b \cdot a^{i+\frac{n}{2}} b=a^{i+\frac{n}{2}} b \cdot a^{i} b, 0 \leq i \leq 2 n$, hence the vertices which are in the form of $\left(a^{i} b, a^{\frac{n}{2}}\right)\left(a^{\frac{n}{2}+i} b, a^{\frac{n}{2}}\right), 0 \leq i \leq 2 n$ are also adjacent to each other. Thus, there is a sequence of complete components of $K_{3}$ graph, since each three commuting vertices are adjacent to each other and $\left|V\left(\Gamma_{\Omega}^{G C}\right)\right|=\frac{3 n}{2}$, thus there are $\frac{n}{2}$ complete components of $K_{3}$ graphs. The results then follow.

Note that if $G$ is a dihedral group of order $2 n$ where $n$ is odd, then the generalized commuting graph, $\Gamma_{\Omega}^{G C}$, cannot be computed, as there will be no set $\Omega$ such that $|a|=|b|=2$ and $a b=b a$, as the center of $G$ will be the trivial center.

The following example illustrates the generalized commuting graph of dihedral group of order 16 .

Example 1: Let $G$ be a dihedral group of order 16 i.e. $G \cong\left\langle a, b: a^{8}=b^{2}=1, a b=b a^{-1}\right\rangle$. Then, $\Gamma_{\Omega}^{G C}=\bigcup_{i=1}^{2^{n-2}} K_{3}$. Solution: Based on Theorem 1, the elements of $\Omega$ are given as follows: There are eight elements in the form of $\left(a^{\frac{n}{2}}, a^{i} b\right), 0 \leq i \leq 2 n$. These elements are $\left(a^{7} b, a^{4}\right),\left(a^{6} b, a^{4}\right),\left(a^{5} b, a^{4}\right),\left(a^{4} b, a^{4}\right),\left(a^{3} b, a^{4}\right)$, $\left(a^{2} b, a^{4}\right),\left(a b, a^{4}\right),\left(b, a^{4}\right)$. There are four elements in the form of $\left(a^{i} b, a^{\frac{n}{2}+i} b\right), 0 \leq i \leq 2 n$. These elements are $\left(a^{3} b, a^{7} b\right),\left(a^{2} b, a^{6} b\right),\left(a b, a^{5} b\right),\left(b, a^{4} b\right)$. Now by applying Definition 2 and Theorem 4 , there are $\frac{8}{2}=4$ complete components of graph which are computed as follows: If $i=0$, the vertices $\left(b, a^{4}\right),\left(a^{4} b, a^{4}\right)$ and $\left(b, a^{4} b\right)$ are adjacent to each other, since they commute with each other. Thus, there is a complete component of $K_{3}$ graph. Similarly, when $i=1,2$ and 3 there are three other complete components of $K_{3}$ i.e. $\Gamma_{\Omega}^{G C}=\bigcup_{i=1}^{4} K_{3}$.

Figure 1 illustrates the generalized commuting graph of the dihedral group of order 16 in Example 1. 

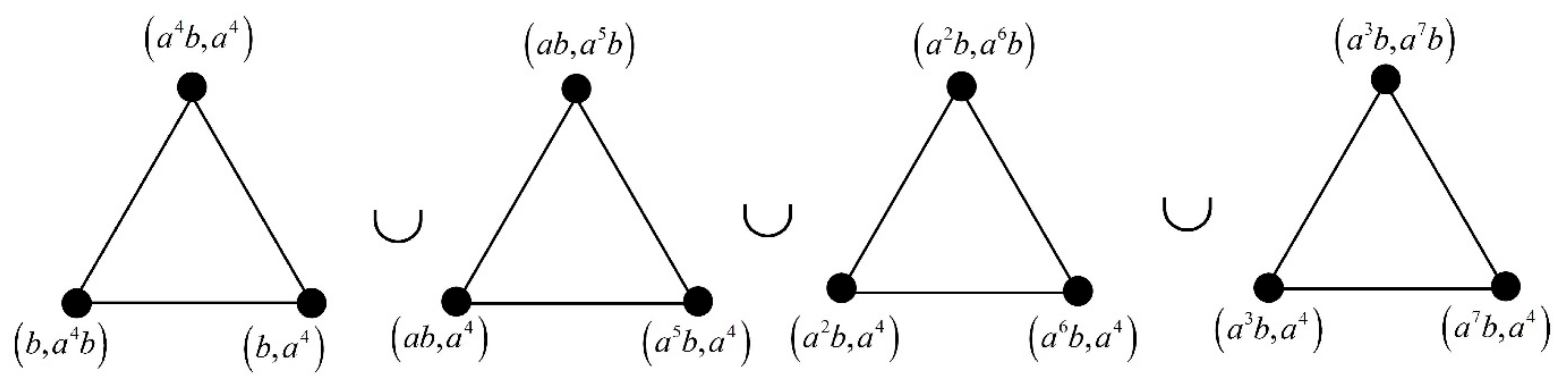

Figure 1. Generalized commuting graphs of the dihedral group of order 16

Next, the generalized commuting graph of the semi-dihedral groups and quasi-dihedral groups are computed, starting with the semi-dihedral groups.

Theorem 5: Let $G$ be a semi-dihedral group, $G \cong\left\langle a, b: a^{2^{n}}=b^{2}=1, a b=b a^{2^{n-1}-1}\right\rangle$, where $n \geq 3$. Let $\Omega$ be a non-empty subset of $G \times G$. Then, $\Gamma_{\Omega}^{G C}=\bigcup_{i=1}^{2^{n-2}} K_{3}$.

Proof: Recall from Theorem 2, $|\Omega|=3\left(2^{n-2}\right)$. Based on Definition 2, the number of vertices in $\Gamma_{\Omega}^{G C}$ is $3\left(2^{n-2}\right)$ i.e. $\left|V\left(\Gamma_{\Omega}^{G C}\right)\right|=3\left(2^{n-2}\right)$. Now two vertices $\omega_{1}, \omega_{2} \in \Omega$ are adjacent if $\omega_{1} \omega_{2}=\omega_{2} \omega_{1}$. It follows that, the vertices which are in the form of $\left(a^{i} b, a^{2^{n-1}+i} b\right), 0 \leq i \leq 2 n$ where $i$ is even are adjacent to the vertices $\left(a^{i} b, a^{\frac{n}{2}}\right), 0 \leq i \leq 2 n$ where $i$ is even. The same is true for vertices that are in the form of $\left(a^{i} b, a^{2^{n-1}+i} b\right)\left(a^{2^{n-1}+i} b, a^{\frac{n}{2}}\right), 0 \leq i \leq 2 n$ where $i$ is even. Since $a^{i} b \cdot a^{2^{n-1}+i} b=a^{2^{n-1}+i} b \cdot a^{i} b, 0 \leq i \leq 2 n, i$ is even, hence the vertices which are in the form of $\left(a^{i} b, a^{\frac{n}{2}}\right),\left(a^{2^{n-1}+i} b, a^{\frac{n}{2}}\right), 0 \leq i \leq 2 n$ where $i$ is even are also adjacent to each other. Thus, there is a sequence of complete components of $K_{3}$ graphs, since each three commuting vertices are adjacent to each other and $\left|V\left(\Gamma_{\Omega}^{G C}\right)\right|=3\left(2^{n-2}\right)$, thus there are $2^{n-2}$ complete components of $K_{3}$ graphs. The results then follow.

Figure 2 illustrates the generalized commuting graph of the semi-dihedral group in general. 


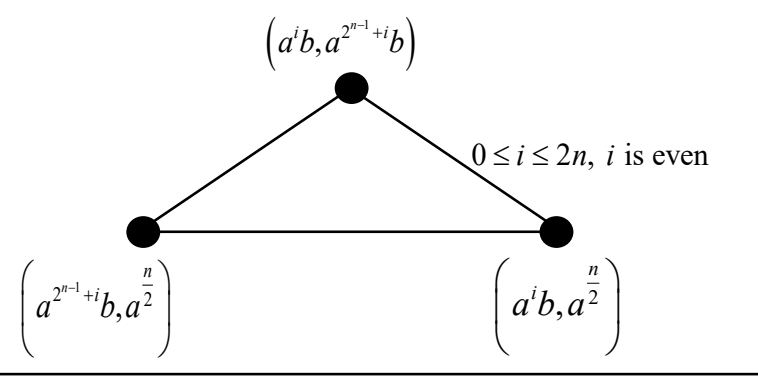

Figure 2. Generalized commuting graph of the semi-dihedral group in general

Next, the generalized commuting graphs of a semi-dihedral group of order 16 are illustrated in the following.

Example 2: Let $G$ be a semi-dihedral group, $G \cong\left\langle a, b: a^{2^{n}}=b^{2}=1, a b=b a^{2^{n-1}-1}\right\rangle$, where $n=3$. Then $\Gamma_{\Omega}^{G C}=K_{3} \cup K_{3}$.

Solution: Based on Theorem 2, the number of elements in $\Omega$ is six, represented as follows: $\left(a^{6} b, a^{2} b\right)$, $\left(a^{4} b, b\right),\left(a^{6} b, a^{4}\right),\left(a^{4} b, a^{4}\right),\left(a^{2} b, a^{4}\right)$, and $\left(b, a^{4}\right)$. According to Definition 2 and Theorem 5 , the vertices $\left(a^{6} b, a^{2} b\right),\left(a^{2} b, a^{4}\right)$, and $\left(a^{6} b, a^{4}\right)$ are adjacent to each other, since they commute with each other. Thus, there is a complete component of $K_{3}$ graph, similarly, the vertices $\left(a^{4} b, b\right),\left(a^{4} b, a^{4}\right)$ and $\left(b, a^{4}\right)$ are adjacent to each other. Hence, there is another complete component of $K_{3}$ graph. Therefore $\Gamma_{\Omega}^{G C}=K_{3} \cup K_{3}$.

The generalized commuting graph of semi-dihedral group of order 16 is illustrated in Figure 3.

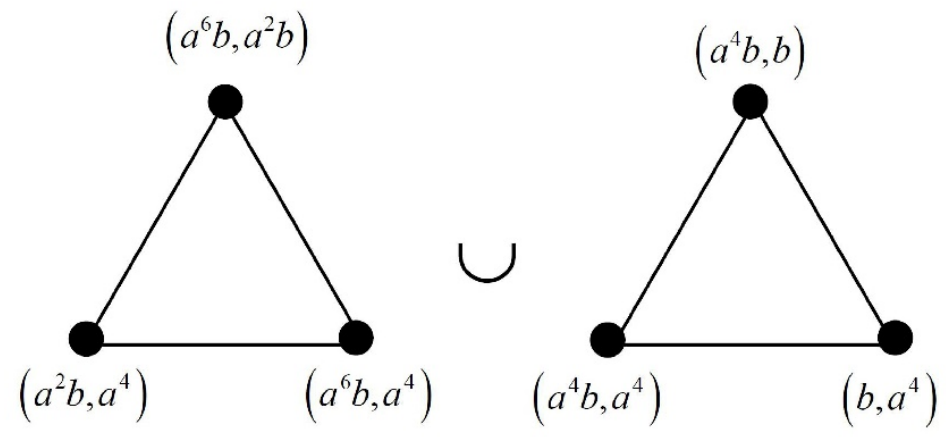

Figure 3. Generalized commuting graphs of the semi-dihedral group of order 16

Our last result is on determining the generalized commuting graph of the quasi-dihedral groups.

Theorem 6: Let $G$ be a quasi-dihedral group, $G \cong\left\langle a, b: a^{2^{n}}=b^{2}=e, a b=b a^{2^{n-1}+1}\right\rangle$. Let $\Omega$ be a nonempty subset of $G \times G$. Then $\Gamma_{\Omega}^{G C}=K_{3}$.

Proof: Recall from the proof of Theorem 3, $|\Omega|=3$. Based on Definition 2, the number of vertices in 
$\Gamma_{\Omega}^{G C}$ is 3, i.e $\left|\Gamma_{\Omega}^{G C}\right|=3$. Now two vertices $\omega_{1}, \omega_{2} \in \Omega$ are adjacent if $\omega_{1} \omega_{2}=\omega_{2} \omega_{1}$. It follows that, the vertices which are in the form of $\left(a^{2^{n-1}}, a^{2^{n-1} i} b\right), 0 \leq i \leq 2^{n}$ are adjacent to the vertex $\left(a^{2^{n-1}} b, b\right)$. Since there are two vertices which are in the form of $\left(a^{2^{n-1}}, a^{2^{n-1} i} b\right), 0 \leq i \leq 2^{n}$. Thus, $\Gamma_{\Omega}^{G C}$ consists of one complete component of $K_{3}$ graph.

Example 3: Suppose $G$ is a quasi-dihedral group, $G \cong\left\langle a, b: a^{2 n}=b^{2}=\varepsilon, a b=b a^{2^{2 n-1}+1}\right\rangle$, where $n=4$. Then $\Gamma_{\Omega}^{G C}=K_{3}$.

Solution: Based on Theorem 3, the number of elements of the set $\Omega$ is three, which can be represented as follows: two elements in the form of $\left(a^{2^{n-1}}, a^{2^{n-1} i} b\right), 0 \leq i \leq 2^{n}$, and one element of the form $\left(a^{2^{n-1}} b, b\right)$. Next, when $n=4$, the elements can be listed as $\left(a^{8}, b\right),\left(a^{8}, a^{8} b\right),\left(a^{8} b, b\right)$. According to Definition 2 and Theorem 6, these elements are the vertices of the generalized commuting graph and they are connected (adjacent) to each other. Thus, there is a complete component of $K_{3}$ graph. Therefore, $\Gamma_{\Omega}^{G C}=K_{3}$.

The generalized commuting graph of quasi-dihedral group of order 16 is illustrated in Figure 4.

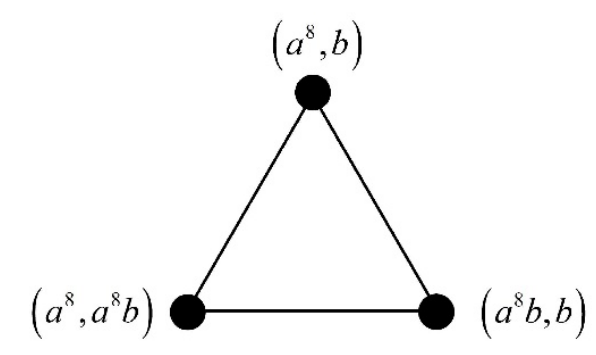

Figure 4. Generalized commuting graph of quasi-dihedral group of order 16

\section{Clique and chromatic numbers of the generalized commuting graphs}

In the previous subsection, it was found that the generalized commuting graph of dihedral, semidihedral and quasi-dihedral groups are either a union of $\frac{n}{2}$ complete graphs of three vertices, a union of $2^{n-2}$ complete graph of three vertices and a complete graph of three vertices.

Proposition 2 Suppose $G$ be a dihedral group of order $2 n$ in which $n$ is even and let $\Omega$ be a nonempty subset of $G \times G$. Thus, the chromatic and clique number of the generalized commuting graph of $G$ is three.

Proof: From Theorem 1, the generalized commuting graph of $G$ is a union of $\frac{2}{n}$ complete graphs 
of three vertices, $\Gamma_{\Omega}^{G C}=\bigcup_{i=1}^{\frac{n}{2}} K_{3}$. Since the maximum size of the set of vertices in any complete graph $K_{n}$ is $n$ itself, therefore the clique number for $K_{3}$ is three. Next, since all $n$ vertices are adjacent towards each other, the minimum number needed to colour the vertices so that no two adjacent vertices have the same colour is also $n$. Therefore, the chromatic number for $K_{3}$ is three. The result then follows.

Proposition 3 Suppose $G$ represent the semi-dihedral group of specific order $2 n$ in which $n$ is even and let $\Omega$ be a non-empty subset of $G \times G$. Thus, the chromatic and clique number of the generalized commuting graph of $G$ is three.

Proof: From Theorem 2, the generalized commuting graph of $G$ is a union of $2^{n-2}$ complete graphs of three vertices, i.e. $\Gamma_{\Omega}^{G C}=\bigcup_{i=1}^{2^{n-2}} K_{3}$. Since the maximum size of the set of vertices in any complete graph $K_{n}$ is $n$ itself, therefore the clique number for $K_{3}$ is three. Next, since all $n$ vertices are adjacent towards each other, the minimum number needed to colour the vertices so that no two adjacent vertices have the same colour is also $n$. Therefore, the chromatic number for $K_{3}$ is three. The result then follows.

Proposition 4 Suppose $G$ represent the quasi-dihedral group of specific order $2 n$ in which $n$ is even and let $\Omega$ be a non-empty subset of $G \times G$. Thus, the chromatic and clique number of the generalized commuting graph of $G$ is three.

Proof: From Theorem 3, the generalized commuting graph of $G$ is a complete graph of three vertices, i.e. $\Gamma_{\Omega}^{G C}=K_{3}$. Next, since all $n$ vertices are adjacent towards each other, the minimum number needed to colour the vertices so that no two connected (adjacent) vertices has the same colour is also $n$. Therefore, the chromatic number for $K_{3}$ is three. The proof then follows.

Table 1. The Chromatic and Clique Numbers of Generalized Commuting Graphs

\begin{tabular}{lccc}
\hline Groups & Number of component of $K_{3}$ & Chromatic number & Clique number \\
\hline Dihedral group & 4 & 3 & 3 \\
Semi-dihedral group & 2 & 3 & 3 \\
Quasi-dihedral group & 1 & 3 & 3 \\
\hline
\end{tabular}

\section{Conclusions}

This study proposed a new graph referred to as generalized commuting graphs. Based on the definition, some related graph properties are presented, in which clique and chromatic numbers were specifically provided. Finally, the generalized commuting graphs of the dihedral group, the semidihedral group and the quasi-dihedral group were also determined. It was found that the generalized commuting graph for these three groups is complete component of $K_{3}$ graph. 


\section{Acknowledgments}

The authors would like to thank reviewers for their time in reviewing and feedback for this paper.

\section{References}

[1] J. Bondy and G. Murty, 1982. Graph Theory with Application, $5^{\text {th }}$ ed. North Holland, Boston, New York.

[2] C. Godsil and G. Royle. 2001. Algebraic Graph Theory, $5^{\text {th }}$ ed. Springer, Boston New York.

[3] R. Brauer, and K. Fowler, "On groups of even order," Ann Math, vol. 62, no. 3, pp. 565-583, 1955.

[4] G. S. Singh, 2010. Graph Theory, PHI Private Learning Limited, New Delhi.

[5] G. Chartrand, L. Lesniak and P. Zhang, 2010. Graphs and Diagraphs, CRC Press, Boca Raton.

[6] Y.Segev, "The commuting graph of minimal nonsolvable groups," Geomtriae Dedicata, vol. 88, pp. 55-66, 2001.

[7] D. Bundy, "The connectivity of commuting graphs," Journal of Combinatorial Theory, Series A, vol. 113, no. 9951007, 2006.

[8] A. Iranmanesh, and A. Jafarzadeh, "On the commuting graph associated with the symmetric and alternating groups," Journal of Algebra and Its Applications, vol. 7, no. 1, 129146, 2008.

[9] C. Bates, D. Bundy, S. Hart, and P. Rowley, "A note on commuting graphs for symmetric groups," The Electronic Journal of Combinatorics, vol. 16, no. 1, 2009.

[10] T. Chelvam, and L. R. S. Selvakumar, "Commuting graphs on dihedral group," Journal of Mathematics and Computer Science, vol. 2, no. 2, pp. 402-406, 2011.

[11] R. Raza, and S. Faizi, "Commuting graphs of dihedral types groups," Applied Mathematics E-Notes, vol. 13, pp. 221-227, 2013.

[12] C. Parker, "The commuting graph of soluble," Bulleting of London Mathematical Society, vol. 45, no. 4, pp. 839848, 2013.

[13] A. Azimi, A. Erfanian, and M. D. G. Farrokhi, "The Jacobson graph of commutative rings," Journal of Algebra and Its Applications, vol. 12, no. 3, pp. 1250179, 2013.

[14] A. Azimi, A. Erfanian, and M. D. G. Farrokhi, "Isomorphism between Jacobson graphs," Rendiconti del Circolo Matematico di Palermo, vol. 63, no. 2, pp. 277-286, 2014.

[15] A. Azimi, and M. D. G. Farrokhi, "Cycles and paths in Jacobson graphs," Ars Combinatoria, vol. 134, pp. 61-74, 2017.

[16] H. Ghayour, A. Erfanian, and A. Azimi, "Some results on the Jacobson graph of a commutative ring," Rendiconti del Circolo Matematico di Palermo Series 2, vol. 67, no. 1, pp. 33-41, 2018.

[17] S. Akbari, M. Habibi, A. Majidinya and R. Manaviyat, "The inclusion ideal graph of rings," Communications in Algebra, vol. 43, no. 6, pp. 2457-2465, 2015.

[18] A. Das, and D. Nongsiang, "On the genus of the commuting graphs of finite non-abelian groups," International Electronic Journal of Algebra, vol. 19, pp. 91-109, 2016.

[19] N. Zaid, N. H. Sarmin and S. N. A. Zamri, "A Variant of Commutativity Degree and Its Generalized Conjugacy Class Graph," Advanced Science Letters, vol. 24, no. 6, pp. 4429-4432, 2018.

[20] N. Zaid, N. H. Sarmin and S. M. S. Khasraw, "On the probability and graph of some finite rings of matrices," AIP Conference Proceedings, vol. 2266, pp. 060010, 2020.

[21] N. Zaid, N. H. Sarmin and S. M. S. Khasraw, "Probabilistic characterizations of some finite rings of matrices and its zero divisor graph," Southeast Asian Bulletin of Mathematics, vol. 44, no. 6, pp. 859-866, 2020.

[22] V. V. Swathi and M. S. Sunitha, "Square graphs of finite groups," AIP Conference Proceedings, vol. 2336, pp. 050014, 2021.

[23] M. A. El-Sanfaz, N. H. Sarmin, and S. M. S. Omer, "On the probability that a group element of the dihedral groups fixes a set," International Journal of Applied Mathematics and Statistics, vol. 52, no. 1, pp. 1-6, 2014.

[24] J. A. Gallian. 2002. Contemporary Abstract Algebra, $5^{\text {th }}$ ed. Houghton Mifflin Company, Boston, New York,

[25] M. A. El-Sanfaz, N. H. Sarmin, and S. M. S. Omer, "On the probability that a group element fixes a set and its generalized conjugacy class graph," International Journal of Mathematical Analysis, vol. 9, no. 4, pp. 161-167, 2015. 